\title{
MITTEILUNGEN
}

\section{Leben nach dem Mandat: Wie Abgeordnete den Ausstieg aus dem Bundestag bewältigen. Eine Veranstaltung der DVParl am 17. Dezember 2008 in Berlin}

Die Abgeordnetenforschung als ein Teil der Elitenforschung befasst sich vornehmlich mit dem Zugang zum politischen Amt, seiner Ausübung, der Einstellung der Amtsträger und dem Verlauf ihrer politischen Karrierewege. ${ }^{1}$ Studien über den Verbleib von ausgeschiedenen Berufspolitikern bilden hingegen nur selten den ausschließlichen Forschungsgegenstand.

Dieser Thematik stellte sich die Deutsche Vereinigung für Parlamentsfragen in einer Veranstaltung am 17. Dezember 2008. Eingeladen war Maria Kreiner (Universität Osnabrück), ihre umfangreiche Forschung über den Verbleib ausgeschiedener Abgeordneter vorzustellen. ${ }^{2}$ Da die Referentin erkrankt war, verlas Heinrich Oberreuter (Universität Passau, Akademie für Politische Bildung Tutzing) die Befunde Kreiners. Nils Diederich (Freie Universität Berlin) kommentierte die Veranstaltung.

Kreiners Studie basiert auf Interviews mit 38 ehemaligen Bundestagsabgeordneten. Die 1994 oder 1998 ausgeschiedenen Parlamentarier wurden von der Verfasserin über ihre Situation nach dem Ausscheiden aus dem Bundestag befragt. Darunter befanden sich sowohl ehemalige Abgeordnete, die nicht wieder kandidierten, als auch MdBs, die nicht wiedergewählt wurden. In die Untersuchung wurden Abgeordnete aller im Bundestag vertretenen Parteien einbezogen, Männer und Frauen, in West- und Ostdeutschland sozialisierte. Die Ergebnisse des beruflichen und politischen Verbleibs wurden anhand dreier Thesen vorgestellt: (1) Ausgeschiedene Berufspolitiker werden von ihrer Partei mit Posten versorgt. (2) Ausgeschiedene Berufspolitiker finden mit ihrer Qualifikation problemlos eine gut bezahlte Stelle. (3) Wer einmal Berufspolitiker war, hat für den Rest seines Lebens materiell und von seinem Ansehen her ausgesorgt.

(1) Die These, dass ausgeschiedene Berufspolitiker durch die Partei mit Posten versorgt werden, muss relativiert und differenziert werden. Die Rolle der Partei für einen beruflichen Wiedereinstieg wurde von den Befragten nahezu einstimmig negativ beurteilt. Sowohl folgenlos gebliebene Unterstützungsbekundungen als auch schlichte Ignoranz bis hin zu

1 Vgl. Heinz Rausch, Der Abgeordnete. Idee und Wirklichkeit, München 1973; Dietrich Herzog, Politische Karrieren. Selektion und Professionalisierung politischer Führungsgruppen, Opladen 1975; John R. Hibbing, Legislative Careers. Why and How We Should Study Them, in: Legislative Studies Quarterly, 24. Jg. (1999), H. 2, S. 149 - 171; Jens Borchert / Klaus Stolz, Die Bekämpfung der Unsicherheit: Politikerkarrieren und Karrierepolitik in der Bundesrepublik Deutschland, in: PVS, 44. Jg. (2003), H. 2, S. 148 - 173; Heinrich Best / Stefan Jahr, Politik als prekäres Beschäftigungsverhältnis: Mythos und Realität der Sozialfigur des Berufspolitikers im wiedervereinten Deutschland, in: ZParl, 37. Jg. (2006), H. 1, S. 63 - 79.

2 Maria Kreiner, Amt auf Zeit. Eine Verbleibsstudie über ehemalige Bundestagsabgeordnete, Baden-Baden 2006; dies., Amt auf Zeit. Eine explorative Studie zum beruflichen und politischen Verbleib ehemaliger Bundestagsabgeordneter, in: ZParl, 38. Jg. (2007), H. 2, S. $261-276$. 
Begegnungsvermeidungen fanden sich im Verhaltensrepertoire der Parteien gegenüber den ausgeschiedenen Bundestagsmitgliedern. Insbesondere Befragte, die ihr Mandat unverhofft und durch eine Wahlniederlage verloren hatten, die sich nicht auf das Ausscheiden vorbereiten konnten und damit eine Unterstützung ihrer Partei begrüßt hätten, fühlten sich im Stich gelassen. Umgekehrt wurden die Befragten, die freiwillig auf ihr Mandat verzichtet hatten, um sich aus der aktiven Berufspolitik zurückzuziehen, mit Nachdruck von ihrer Partei gedrängt, für andere politische Ämter zu kandidieren. Dieser Umstand hinderte die Betroffenen zum Teil sogar daran, eine Erwerbsarbeit außerhalb der Politik anzunehmen. Die Untersuchungsergebnisse lassen sich mit der Art des Ausscheidens der Befragten erklären. Der Mandatsverlierer - also derjenige, der vorwiegend von der Partei ignoriert worden ist - hatte offenbar weder bei der Parteispitze noch bei der Parteibasis oder bei den Wählern entsprechend Rückhalt gefunden, um das Mandat halten zu können. Hingegen waren die von der Partei Umworbenen alle aus einer stabilen politischen Position freiwillig ausgeschieden und besaßen deshalb nach wie vor das Potenzial, auch in andere Ämter gewählt zu werden. Die These, dass ausgeschiedene Abgeordnete von ihrer Partei mit Posten versorgt werden, kann nur in Ausnahmefällen bestätigt werden, denn die Parteien verfügen in der Regel nicht über stabile Auffangnetze.

(2) Befragte beschreiben, dass sie sich während ihrer Bundestagszeit vom normalen Leben entfremdet hätten und abgehoben seien. Sie begründeten dies mit den Privilegien, die notwendigerweise mit dem Mandat verbunden sind, und der repräsentativen Funktion, die sie einnehmen. Diese brachte es mit sich, dass sie von ihrem sozialen Umfeld in einer herausgehobenen Weise behandelt wurden. Dieser mehr oder minder lange andauernde $\mathrm{Zu}-$ stand stellte für manche eine Hürde für den beruflichen Wiedereinstieg dar. Durch die erworbenen Fähigkeiten als Parlamentarier waren sie für gehobene Berufspositionen qualifiziert. Deshalb hatten manche Schwierigkeiten, in Erwerbspositionen der mittleren Ebene einzusteigen; sie galten als überqualifiziert. Am Arbeitsplatz wurden sie nicht mehr als Gleiche akzeptiert und konnten sich nur schwer in ein Team integrieren. Die These, dass Berufspolitiker mit ihrer Qualifikation problemlos eine gut bezahlte Stelle finden, ist demnach nicht haltbar.

(3) Fälle von ausgeschiedenen Bundestagsabgeordneten, die nach dem Mandatsverlust in eine wirtschaftliche Notlage geraten sind, waren sowohl bei Befragten aus Ost- als auch aus Westdeutschland anzutreffen. Wie einzelne Interviewpartner berichteten, sind aber insbesondere Ostabgeordnete von wirtschaftlichen Notlagen nach einem Mandatsverlust betroffen. Insgesamt kann daher die These, dass, wer einmal Berufspolitiker war, für den Rest seines Lebens materiell ausgesorgt habe, keine uneingeschränkte Gültigkeit beanspruchen.

Die von Kreiner dokumentierten Daten und Einschätzungen zum Verbleib ehemaliger Bundestagsabgeordneter sind nicht repräsentativ. Sie vermitteln aber ein realistisches Bild der ausgeschiedenen Berufspolitiker und zeigen, dass mit dem Amt auf Zeit Risiken für den nachpolitischen Lebensweg verbunden sind. In der anschließenden Diskussion wurden daraus resultierende Fragen zum vorparlamentarischen Rekrutierungsbereich, dem Versorgungsschutz und dem Berufsbild des Abgeordneten im Allgemeinen besprochen.

Bei der Betrachtung des Rekrutierungsbereichs und der daraus resultierenden berufssoziologischen Zusammensetzung des Bundestages stellte Diederich die These in den Mittelpunkt, dass die Abgangsbedingungen den Zugang zum Amt bestimmen und damit das von Mandatsaspiranten vorhergesehene und als inakzeptabel beurteilte Risiko des existenziellen Scheiterns wesentlich für die berufssoziologische Zusammensetzung des Deutschen Bun- 
destages mitverantwortlich sei. Torsten Wolfgramm berichtete diesbezüglich von seinen Erfahrungen aus der FDP-Fraktion. Nach seinem Eindruck ist der Anteil der Freiberufler gesunken und der von Parlamentariern, die aus dem öffentlichen Dienst kommen, angestiegen, denn Freiberufler haben den Nachteil, nur schwer wieder in ihren Beruf zurückkehren zu können. Suzanne S. Schüttemeyer (Martin-Luther-Universität Halle-Wittenberg) verwies darauf, dass die Daten für Bundestagsabgeordnete dies nicht ausweisen. Die Anzahl der Beamten liege im Laufe der Wahlperioden sogar relativ stabil bei circa einem Drittel. Auch habe der Anteil an Freiberuflern eher wieder zugenommen.

Joachim Hörster (MdB, CDU/CSU-Fraktion) machte auf die sich in den letzten Jahren verschlechternden Versorgungsbezüge der Abgeordneten aufmerksam. Die Tatsachen, dass es ehemalige Bundestagsabgeordnete gibt, die nach dem Mandatsverlust zu Sozialhilfeempfängern werden und dass das Übergangsgeld kaum seinem eigentlichen Zweck dient, sollte debattiert werden. Er verwies darauf, dass „es einer funktionierenden Demokratie etwas wert sein muss, Leute zu haben, die vielleicht drei Wahlperioden im Bundestag sind, aber dann auch einen solchen sozialen Status haben, dass man keine Angst davor zu haben braucht, in den Bundestag gewählt zu werden“. Der Abgeordnete Gero Storjohann (CDU/ CSU-Fraktion) unterstrich die Forderung nach einem Alterssicherungssystem für Abgeordnete. Berufspolitiker ließen sich nicht mit Unternehmern vergleichen, die die Möglichkeit haben, sich am Markt neu zu positionieren; sie seien „abhängig von dem Gesamtbild ihrer Partei in der Öffentlichkeit“.

Skeptisch äußerte sich Michael Edinger (Friedrich-Schiller-Universität Jena) zu der These, dass die Abgangsbedingungen einen sehr deutlichen Einfluss auf den Zugang zum Mandat haben. Das würde voraussetzen, dass die Kandidaten, die sich um ein Mandat bemühen, ziemlich gute Kenntnisse darüber haben, was die Ehemaligen tun. Dass beim Einstieg ins Parlament schon darüber nachgedacht wird, was eigentlich am Ende steht, bestätigte Storjohann jedoch aus seiner Erfahrung. Darüber hinaus waren sich Oberreuter und Diederich einig, dass jeder Kandidat sich „tunlichst überlegen sollte, was daraus wird. Es ist auch die Mitverantwortung jedes Einzelnen, seine Position hinterher zu finden“. Ratsam ist, weiterhin Kontakt zu seiner beruflichen Tätigkeit zu halten.

Joachim Linck (Friedrich-Schiller-Universität Jena) zog aus Kreiners Ergebnissen Folgerungen für das Berufsbild des Abgeordneten. Berufsabgeordnete kämpften ständig um den Erhalt ihres Mandats, was zur - von Kreiner beschriebenen - Abgehobenheit führe. Aus Lincks Sicht liegt die Ursache für dieses Ergebnis in der Professionalisierung des Abgeordnetenberufs begründet. Durch eine Abkehr vom Berufspolitiker zurück zum Teilzeitabgeordneten könne diese Entwicklung aufgehalten werden. Schüttemeyer und Edinger widersprachen für die Ebene des Bundestages vehement, da hier auf keinen Fall Amateurpolitiker die notwendigen Leistungen erbringen können, die vom Bürger gefordert sind. Linck konkretisierte, dass sein Vorschlag nur für die „Feierabendparlamente“ in den Ländern gelten solle.

Insgesamt räumte der Abend mit Vorurteilen gegenüber ehemaligen Berufspolitikern auf. Die Vorstellung, dass man mit einem Bundestagsmandat für alle Zeit finanziell abgesichert sei, wiesen alle Redner als Klischee aus, das bequem transportiert wird, aber keine Allgemeingültigkeit besitzt. Über den Befund, den Kreiner vorlegte, herrschte Einigkeit. Die Frage, welche Reform Linderung versprechen könnte, bedarf weiterer Diskussionen. 